\title{
Trigeminal sensitivity to contact chemical stimulation: A new method and some results
}

\author{
JAMES D. PRAH and VERNON A. BENIGNUS \\ U.S. Environmental Protection Agency \\ and the University of North Carolina, Chapel Hill, North Carolina
}

\begin{abstract}
Acquisition of knowledge about the chemical senses might be hastened if chemicals or techniques that permitted stimulation of either the common chemical sense, mediated by the trigeminal nerve, or the sense of smell, mediated by the olfactory nerve without concomitant stim. ulation of the other sense, were used. Flowing the nonodorized output of an olfactometer into one naris of subjects while velopharyngeal closure was maintained produced effluent flow from the contralateral naris. To prevent odorous molecules from reaching the olfactory epithelium, the contralateral naris was stimulated by applying diluted and undiluted chemical solutions directly to the septal squamous epithelium downstream from the olfactory mucosa. A series of five geometric dilutions of three chemicals and one saturated solution of a fourth were used. The weakest dilution of each chemical was an adequate olfactory stimulus. Two were known trigeminal stimulants (acetic acid and butanol), and two were rated low in trigeminal stimulatory capacity (phenethyl alcohol and vanillin). The results showed that this method was useful in determining the trigeminal threshold for some chemicals. When this method was used, saturated aqueous solutions of vanillin and phenethyl alcohol were clearly not detectable by the trigeminal receptors. The application of the undiluted vanillin and phenethyl alcohol yielded a clear sensation. This result may imply that there are no pure olfactory stimuli.
\end{abstract}

To some extent, progress in research on the chemical senses has been hampered by the number of chemically sensitive systems in the upper respiratory system. Most lower mammals have four chemoreceptive senses: smell, taste, common chemical, and vomeronasal. This excludes the nervus terminalis and the septal organ, whose sensory capabilities and roles are unknown (Wysocki, 1979). In man, the vomeronasal system may be rudimentary and nonfunctional (Wysocki, 1979). In other animals, the vomeronasal organ is thought to be involved in reproduction and feeding (Bertmar, 1975). While the sense of taste plays a minor role in olfaction, the sense of smell seems to have a larger role in taste. This can be observed in anosmics to whom food no longer tastes the same. In humans, the interactions between the common chemical sense (Parker, 1912), mediated by the trigeminal nerve, and the sense of smell normally prevent the independent study of either sense.

The human nasal cavity is innervated by the ophthalmic and maxillary branches of the trigeminal nerve (Schade, 1966), whose free nerve endings are chemically sensitive, and the olfactory nerve, whose ciliated processes are also chemically sensitive. In stimulating the olfactory receptors, one also stimulates the trigeminal free nerve endings unless steps are taken to avoid concomitant stimulation. The trigeminal nerve may potentiate or have a synergistic effect on olfaction. This was

Requests for reprints should be addressed to James D. Prah, U.S. Environmental Protection Agency, Medical Building C No. 224H, Mason Farm Road, Chapel Hill, North Carolina 27514. illustrated by Cain (1974), who demonstrated an increased magnitude of perceived odor due to a trigeminal component in the absence of obvious irritation.

Progress in the study of the chemical senses may be hastened by the identification of chemicals that stimulate each sensory system independently of the others and is applicable to normal intact subjects. While it is unclear whether a pure olfactory stimulus exists (Tucker, 1971), the use of a pure olfactory stimulant, for example, would eliminate the trigeminal input to the sensation of smell and its apparent inhibitory effect on olfaction (Cain \& Murphy, 1980). The use of an apparently pure trigeminal stimulant such as $\mathrm{CO}_{2}$ (Cain \& Murphy, 1980) would permit the common chemical sense to be studied independently of the olfactory sense.

The human nasal epithelium varies in surface configuration from squamous epithelium anteriorly to pseudostratified columnar epithelium posteriorly. Little is known about the absolute or differential capabilities of the variety of tissue types found in the nasal mucosa. These tissues appear to have a limited capacity for the localization of stimuli and discrimination of sensory modalities (Cauna, 1982). This lack of discriminatory ability is due, perhaps, to the lack of diverse receptor types found in this tissue. It is limited to simple terminal arborizations and apparently has no other receptors such as Pacinian corpuscles or Merckels disks (Cauna, Hinderer, \& Wentges, 1969). Garcia Medina and Cain (1982) have demonstrated that the nasal mucosa is capable of bilateral integration in response to dichorhinic stimulation with carbon dioxide. 
The development of a technique that permits the subject to respond to chemical trigeminal stimulation without having to, by introspection, separate out an olfactory component is described here. The strategy is one that may be useful with normal subjects (1) in the identification of chemicals that are odorous but not trigeminally stimulating, and (2) in the identification of the trigeminal threshold.

\section{METHOD}

\section{Subjects}

The subjects were the two male authors, aged 36 and 45 years, and two female volunteers, aged 28 and 55 years.

\begin{abstract}
Materials
Phenethyl alcohol and vanillin were selected for their apparent lack of trigeminal stimulatory capacity in humans (Doty, 1975; Doty, Brugger, Juos, Orndorff, Snyder, \& Lowry, 1978; Elsberg, Levy, \& Brewer, 1935). Butanol and acetic acid were chosen for their ability to stimulate the trigeminal receptors. Deionized water was used to make a series of five geometric dilutions of three of the stimulants. Delivery of the stimulus to the nasal mucosa was effected in the liquid phase to maintain control over the area stimulated and the delivery of the stimulus. All chemicals were the highest grade obtainable from Fisher Scientific. The strongest solutions of certified butanol, reagent grade acetic acid, and purified grade phenethyl alcohol were 5\% $(50,000 \mathrm{ppm}), 4 \%(40,000 \mathrm{ppm})$, and $1 \%(10,000 \mathrm{ppm})$, respectively. A $1 \%$ solution of phenethyl alcohol was the highest concentration obtainable due to its low solubility in water. To obtain a saturated solution of vanillin, $8 \mathrm{~g}$ were dissolved in $1,000 \mathrm{ml}$ of deionized water. This solution was filtered through a Whatman No. 44 filter to remove any residual crystals. The saturated solution and pure crystalline vanillin were the only levels of this chemical used (see Results). The solutions were kept at room temperature in $125-\mathrm{ml}$ brown glass bottles with glass stoppers. A blank of deionized water was included in the series. The weakest solution of each chemical was detectable by all subjects by sniffing the headspace above the solution.
\end{abstract}

\section{Procedure}

The subject introduced into his left naris, while lying on his left side, the unodorized output of an olfactometer (Benignus \& Prah, 1980a). While maintaining velopharyngeal closure, the airstream thus exited the right naris. Stimulation was effected in liquid phase application to the right septal squamous epithelium. Using this technique, the air flowed in one naris and out the contralateral and stimulated naris, thus preventing the stimulus material from contacting the olfactory epithelium. The air flow was automatically maintained at $2.5 \mathrm{liters} / \mathrm{min}$, and the temperature and relative humidity were $28.6^{\circ} \mathrm{C}$ and $70 \%$, respectively. While this flow was above the detection level for pulses of air at this temperature and humidity (Benignus \& Prah, 1980b), the constant flow adapted out in a matter of seconds. The subject was instructed to breathe slowly and gently through the mouth while maintaining velopharyngeal closure. This technique is similar to the Valsalva maneuver (Mozell, 1971). A vacuum line was placed above the lip and below the stimulated naris to prevent the effluent air from entering the oral cavity. None of the subjects reported tasting the stimuli. This is analogous to the technique used by Mozell, Smith, Smith, Sullivan, and Swender (1969) to prevent odorous stimulation of the nasal chemoreceptors during lingual flavor stimulation.

Direct stimulation of the mucosa innervated by the maxillary branch of the trigeminal nerve endings was obtained by dipping a cotton-tipped applicator into the stimulus solution, removing the excess liquid by gently pressing it against the interior neck of the bottle, and then inserting it under visual guidance into the medial nasal septum, which lies anteroventral to the inferior turbinate. The applicator was gently rolled once in each direction across an area of about $1 \mathrm{~cm}^{2}$ of the nasal mucosa. After the subject indicated a response of "yes" or "no" with hand signs, the stimulated area was gently cleansed with six applicators in a wetdry sequence. An applicator was wetted in deionized water, then the excess water was removed and the stimulated area was wiped and dried with a fresh applicator. This cleansing series was done three times. Each trial took approximately $20 \mathrm{sec}$ to complete. A preliminary study had indicated that no adaptation took place at this rate of stimulation. This procedure was repeated for each of six dilutions five times, in a random order. Two series were obtained on each chemical except vanillin (see Results).

\section{RESULTS}

The results indicate that psychophysical functions and thresholds to liquid phase trigeminal stimulation can be obtained for acetic acid and butanol (Figure 1) using this technique. These obtained trigeminal thresholds were found to be greater than the olfactory thresholds for the same two substances (Prah \& Benignus, 1979). In the earlier study, using sniff bottles, the olfactory thresholds for eight subjects were found to be between $0.025 \%$ and $0.0125 \%$ for acetic acid and between $0.0125 \%$ and $0.00625 \%$ for butanol. No graphs are provided for phenethyl alcohol because three of the four subjects were trigeminally insensitive to the substance. The fourth subject (L.L.B.) developed a hypersensitivity to the procedure and produced a high false-alarm rate. Since none of the subjects was able to detect the highest level of the vanillin solution, no curves are presented for this stimulus.

All of the subjects consented to have undiluted phenethyl alcohol and vanillin crystals applied to the medial septum, which resulted in a clear trigeminal response that was enhanced upon commencement of the cleansing procedure. This sensation was described by the subjects as one of slight burning, itching, or stinging that outlasted the tactile contact of the stimulus presentation.

\section{DISCUSSION}

This technique to stimulate the trigeminally innervated nasal mucosa was demonstrated to be a viable method for obtaining detection thresholds of butanol and acetic acid in the absence of olfactory stimulation in normal subjects. It required that discriminations be made between the common chemical sensation and the temperature and tactile sensations of the stimulus application. As can be seen from the figure, the response rate to the blank of deionized water was very low, indicating that the subjects were able to make these discriminations with ease.

Although it is difficult to compare directly the delivery of the stimulus in the liquid phase with a stimulus delivered in the vapor phase, the use of the liquid phase may have permitted the stimulus to be presented in a more effective manner for two reasons. First, the 


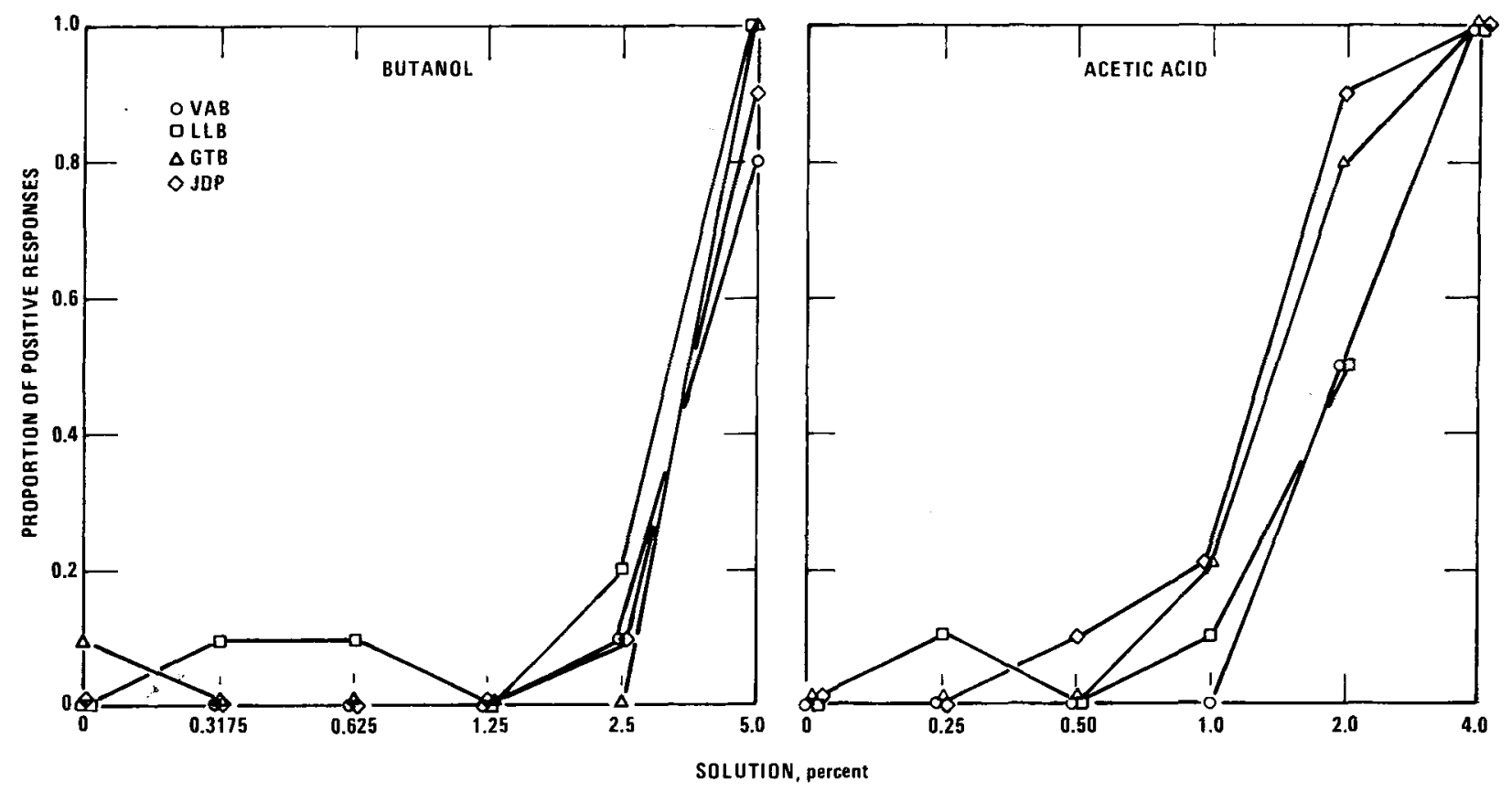

Figure 1. Psychophysical functions for butanol and acetic acid. Each point represents 10 stimulations.

concentrations available may be greater than those available in the vapor phase due to low vapor pressure of some chemicals. Second, a water-borne stimulus directly applied to the mucus will readily dissolve in the watery mucus and thus reach the mucosa more rapidly than an air-borne stimulus that must first penetrate the air-mucus interface before reaching the mucosa. As a result, the estimates of the thresholds derived via this technique will probably be more conservative than those derived using an air-borne stimulus. Thus, if the stimulus is not detectable at these concentrations in liquid, they are probably not detectable at lower concentrations in the vapor phase.

Since neither phenethyl alcohol nor vanillin was detected at aqueous saturation but was clearly detectable when undiluted, the threshold must be between the two. It would be difficult to determine the liquidphase threshold more precisely. Other solvents for these chemicals include ethanol, carbon disulfide, and acetone (Weast, 1970). These solvents are also trigeminal stimulants and would confound the sensation if used to obtain a greater concentration.

The detection of undiluted phenethyl alcohol and vanillin, two substances that were not frequently detected by anosmic subjects (Doty, 1975; Doty et al., 1978), was of particular interest. It may imply that there are no pure olfactory stimuli, or it may imply that if given in high enough concentrations, any chemical is detectable. What tends to militate against the latter conclusion is that the sensation was not immediate. It reflected the time needed for the stimulus to dissolve in the mucus and penetrate the mucosa to the receptors. One may assume that the concentration dissolved in the mucus was saturated and thus would reflect the maxi- mum physiological concentration available to the innervated mucosa.

While it cannot be concluded that there are no pure olfactory stimuli, this technique may prove to be a useful method for screening chemicals, although it must, necessarily, be limited to those that are water soluble. These data and this technique complement olfactory threshold data obtained in an earlier experiment and may be useful in choosing olfactory stimuli levels below the trigeminal threshold.

As mentioned earlier, the sensitivity of the nasal mucosa seems to be limited. It may be of interest to explore further the capabilities of this system. We know it is capable of discriminating levels of chemical stimuli, but we do not know if it is capable of discriminating between chemicals. Researchers studying the chemical senses may be interested in pursuing the question further.

\section{REFERENCES}

Benignus, V. A., \& Prah, J. D. Flow thresholds of nonodorized air through the human naris as a function of temperature and humidity. Perception \& Psychophysics, 1980, 27, 569-573. (a)

Benignus, V. A., \& Prah, J. D. A computer-controlled vapordilution olfactometer. Behavior Research Methods \& Instrumentation, 1980, 12, 535-540. (b)

Bertmar, G. Ecochemical studies on reindeer. In D. A. Denton \& J. P. Coghlan (Eds.), Olfaction and taste V. New York: Academic Press, 1975.

Caln, W. S. Contribution of the trigeminal nerve to perceived odor magnitude. Annals of the New York Academy of Sciences, 1974, 237, 28-34.

CaiN, W. S., \& Murphy, C. L. Interaction between chemoreceptive modalities of odour and irritation. Nature, 1980, 284, 255-257. 
Cauna, N. Blood and nerve supply of the nasal lining. In D. Proctor \& I. Andersen (Eds.), The nose. New York: Elsevier Biomedical Press, 1982.

Cauna, N., Hinderer, K. H., \& Wentges, R. T. Sensory receptor organs of the human nasal respiratory mucosa. American Journal of Anatomy, 1969, 124, 187-210.

Dоту, R. L. Intranasal trigeminal detection of chemical vapors by humans. Physiology \& Behavior, 1975, 14, 855-859.

Doty, R. L., Brugger, W. E., Jurs, P. C., Orndorff, M. A., SNYDER, P. J., \& Lowky, L. D. Intranasal stimulation from odorous volatiles: Psychometric responses from anosmic and normal humans. Physiology Behavior, 1978, 20, 175-185.

Elsaera, C. A., Levy, I., \& Baewer, E. D. The sense of smell. VI. The trigeminal effects of odorous substances. Bulletin of the Neurological Institute of New York, 1935, 4, 270-293.

Garcia Medina, M. R., \& Cain, W. S. Bilateral integration in the common chemical sense. Physiology \& Behavior, 1982, 29, 349-353.

Mozetz, M. M. The chemical senses: II. Olfaction. In J. W. Kling \& L. A. Riggs (Eds.), Experimental psychology. New York: Holt, Rinehart, \& Winston, 1971.

Mozell, M. M., Smith, B. P., Smith, P. E., Sullivan, R. L., \& Swender, P. A technique to occlude the nasal chemore- ceptors during lingual flavor stimulation. Physiology \& Behavior, 1969, 4, 131.

Parker, G. H. The relation of smell, taste and the common chemical sense in vertebrates. Journal of the Academy of Science, Philadelphia, 1912, 15, 221-234.

Prah, J. D., \& Benignus, V. A. Decrements in olfactory sensitivity due to ozone exposure. Perceptual and Motor Skills, 1979, 48, 317-318.

Schade, J. P. The peripheral nervous system. New York: Elsevier, 1966.

Tucken, D. Nonolfactory responses from the nasal cavity: Jacobsen's organ and the trigmenial system. In L. M. Beidler (Ed.), Handbook of sensory physiology (Vol. 4), Chemical senses (Part 1), Olfaction. New York: Springer-Verlag, 1971.

Weast, R. C. (Ed). Handbook of chemistry and physics. Cleveland, Ohio: The Chemical Rubber Co., 1970.

WYsocki, C. J. Neurobehavioral evidence for the involvement of the vomeronasal system in mammilian reproduction. Neuroscience \& Behavioral Reviews, 1979, 3, 301-341.

(Manuscript received February 14, 1983;

revision accepted for publication September 30, 1983.) 